\title{
hsa-miR-15a exerts protective effects against osteoarthritis by targeting aggrecanase-2 (ADAMTS5) in human chondrocytes
}

\author{
XIN LU, JIN LIN, JIN JIN, WENWEI QIAN and XISHENG WENG \\ Department of Orthopaedic Surgery, Peking Union Medical College Hospital, Beijing 100730, P.R. China
}

Received May 5, 2015; Accepted December 17, 2015

DOI: $10.3892 / \mathrm{ijmm} .2015 .2446$

\begin{abstract}
The aim of the present study was to examine the expression levels and role of hsa-miR-15a in osteoarthritis (OA), as well as the associated mechanisms. The expression levels of hsa-miR-15a and A disintegrin-like and metalloprotease (reprolysin type) with thrombospondin type 1 motif, 5 (ADAMTS5, also known as aggrecanase-2) were measured by reverse transcription-quantitative polymerase chain reaction (RT-qPCR) in both OA and normal chondrocytes. hsa-miR-21 mimics or antisense oligonucleotides (ASO) were co-transfected into the chondrocytes to examine the effects on the putative binding sites compared with the negative control (NC)-mimics or NC-ASO. The relative ADAMTS5 mRNA and protein levels were measured by RT-qPCR and western blot anlaysis, respectively. Moreover, after inhibiting the expression of hsa-miR-15a and ADAMTS5 by ASO and small interfering RNA (siRNA), respectively, the amounts of proteoglycan and collagen in the cellular matrix and medium were determined. Additionally, the expression levels of collagen II were measured by western blot analysis. hsa-miR-15a expression was downregulated, but ADAMTS5 expression was upregulated in the human OA chondrocytes compared to the normal chondrocytes. Luciferase reporter assay confirmed that the hsa-miR-15a binding site was in the ADAMTS5 gene 3'-untranslated region (3'-UTR), and ADAMTS5 was negatively regulated by hsa-miR-15a. The downregulation of hsa-miR-15a decreased the aggregation of proteoglycan and the collagen content, but increased the release of proteoglycan and collagen; total collagen production was significantly lower, and collagenase activity was markedly higher. The downregulation of ADAMTS5 increased the aggregation of proteoglycan and the collagen content, but decreased the release of proteoglycan and collagen, along with total collagen production. Moreover, collagenase activity was markedly lower. The findings of our study suggest that
\end{abstract}

Correspondence to: Dr Xin Lu, Department of Orthopaedic Surgery, Peking Union Medical College Hospital, 1 Shuai Fu Yuan, Wang Fu Jing Street, Beijing 100730, P.R. China

E-mail: luxin8899@126.com

Key words: osteoarthritis, hsa-miR-15a, collagen, aggrecanase-2, proteoglycan
hsa-miR-15a exerts protective effects against OA by targeting ADAMTS5 in human chondrocytes.

\section{Introduction}

Osteoarthritis (OA) is one of the most common degenerative joint disorders, and it affects elderly individuals in particular (1-3). It is characterized by the progressive loss of the cartilage matrix, a reduction in bone mass, the destruction of articular cartilage and osteophyte formation $(4,5)$. The prevalence of this disease has been reported as continuing to increase, and this brings with it an enormous financial burden and related impairment of quality of life $(6,7)$. However, to date, limited available diseasemodifying treatment options for patients with OA are available due to the uncertain etiology of the disease (8). Currently, the only registered systemic oral drug therapy for OA is analgesics or anti-inflammatory agents to relieve symptoms $(8,9)$.

The functional roles of microRNAs (miRNAs or miRs) in OA have increasingly attracted attention (10-12). miRNAs are short, small non-coding RNAs molecules that are involved in various human diseases $(13,14)$. miRNAs regulate gene expression by repressing messenger RNA (mRNA) translation and promoting mRNA degradation (15). Previous studies have confirmed that a number of miRNAs, such as hsa-miR-21, hsamiR-23a, hsa-miR-24, hsa-miR-27, hsa-miR-93, hsa-miR-100, hsa-miR-140, hsa-miR-145, hsa-miR-146a and hsa-miR-675 are associated with the development of OA (16-21). In addition, it has been previously demonstrated that A disintegrin-like and metalloprotease (reprolysin type) with thrombospondin type 1 motif, 5 (ADAMTS5, also known as aggrecanase-2) is involved in the development of OA (22). Of the ADAMTS family, ADAMTS4 and 5 are known to be the most efficient aggrecanases, and have been regarded as the most likely candidates for OA pathogenesis (23). Moreover, a study using animals indicated that the deletion of ADAMTS5 in mice with OA can prevent early aggrecan depletion and cartilage destruction (24). In addition, in another previous study, Matsukawa et al suggested that miR-125b regulates the expression of ADAMTS4 in OA chondrocytes (25).

However, to date, and to the best of our knowledge, there are limited studies available investigating the effects of hsa-miR-15a on OA, and the question of whether hsa-miR-15a regulates the expression of ADAMTS5 remains unanswered. Therefore, in the present study, we aimed to examine the effects of hsa-miR-15a on OA, as well as the associated mechanisms. 


\section{Materials and methods}

Specimen selection. Femoral condyles or tibial plateaus were selected as specimens. Human OA cartilage was harvested from patients who underwent total knee replacement surgery (10 males and 6 females; mean age, $70 \pm 4$ years) at the Department of Orthopaedic Surgery, Peking Union Medical College Hospital (Beijing, China). OA was diagnosed according to the clinical and radiological evaluation criteria published by the American College of Rheumatology (ACR) (26). In addition, none of the patients from whom the samples were collected had received intra-articular steroid therapy in the 3 months prior to the surgery. Healthy (control) articular cartilage was obtained from donors who had died (by car accident or due to cardiovascular and cerebrovascular diseases such as severe heart failure, or malignant tumors), within $12 \mathrm{~h}$ of death ( 8 males and 5 females; mean age, $67 \pm 5$ years). The normal healthny individuals had no previous history of joint disorder. This study was approved by the Medical Ethics Committee of Peking Union Medical College Hospital (Beijing, China) and informed consent was acquired from all participants.

Cell culture and transient transfection. After collecting the cartilage specimens, the tissues were diced and digested in $1.5 \mathrm{mg} / \mathrm{ml}$ collagenase type 2 (CLS-2; Worthington, Lakewood, NJ, USA) in Dulbecco's modified Eagle's medium (DMEM; Invitrogen, Carlsbad, CA, USA) for $16 \mathrm{~h}$ at $37^{\circ} \mathrm{C}$. The DMEM solution was supplemented with $10 \%$ fetal bovine serum (FBS), $100 \mathrm{U} / \mathrm{ml}$ penicillin and $100 \mu \mathrm{g} / \mathrm{ml}$ streptomycin (all from Sigma, St. Louis, MO, USA). The isolated cells were then passed through a $100-\mu \mathrm{m}$ cell strainer (Falcon; Becton-Dickinson Labware GmbH, Heidelberg, Germany), pelleted and washed with medium. The cultures were maintained in DMEM containing $10 \%$ FBS in a humidified incubator containing $5 \% \mathrm{CO}_{2}$ at $37^{\circ} \mathrm{C}$.

The cells were subsequently seeded in a 48 -well prior to transfection. After post-culturing for $18-24 \mathrm{~h}$, when the density of the chondrocytes had reached $70 \%$, the cells were transfected using Lipofectamine 2000 (Invitrogen) according to a previously described method (27). In the present study, hsa-miR-15a mimics, pcDNA3/enhanced green fluorescent protein (EGFP)-ADAMTS5-3'-untranslated region (3'-UTR), antisense oligonucleotides (ASO)-hsa-miR-15a, ASO-NC (negative control), pcDNA3/EGFP-ADAMTS5-3'-UTR mutant and pDsRed2-N1, along with the plasmids were constructed and synthesized by Sangon Biotech Co., Ltd. (Shanghai, China) based on standard protocols (28). Furthermore, ADAMTS5 expression was suppressed using the small interfering RNA (siRNA) synthesized by Sangon Biotech Co., Ltd. in both human OA and normal chondrocytes. A negative control (siNC) was also performed concurrently on each specimen. The sequence of the ADAMTS5 siRNA was 5'-AAGAUAAGCG CUUAAUGUCUU-3'. Cells were collected $48 \mathrm{~h}$ after transfection for subsequent assays.

Analysis of miRNA expression. After post-culturing for $24 \mathrm{~h}$, total RNA was extracted from the cells using an miRNA Isolation kit (Ambion, Austin, TX, USA) according to the manufacturer's instructions. The relative expression of levels miRNAs (specific for hsa-miR-15a) were determined using TaqMan ${ }^{\circledR}$ MicroRNA assays (Applied Biosystems, Foster City,
CA, USA) with the comparative $2^{-\Delta \Delta C T}$ method. Total RNA was reverse transcribed into complementary DNA (cDNA) using MuLV reverse transcriptase (Life Technologies, Carlsbad, CA, USA), followed by quantification using the QuantiTect SYBRGreen real-time-polymerase chain reaction kit (Qiagen SA, Hilden, Germany). Primers for miR-15a were designed and obtained from the TaqMan miRNA assays. U6 snRNA (Applied Biosystems) was used as a loading control for miRNA expression. The primer sequence for stem-loop RT-PCR hsa-miR-15a was 5'-CTCAACTGGTGTCGTGGAGTCGGC AATTCAGTTGAGCACAAACC-3'. The following primers were used for PCR: hsa-miR-15a forward, 5'-ACACT CCAGCTGGGTAGCAGCACATAATGG-3' and reverse, 5'-TGGTGTCGTGGAGTCG-3'; U6 forward, 5'-CTCGCTT CGGCAGCACA-3' and reverse, 5'-AACGCTTCACGAA TTTGCGT-3'.

Reverse transcription-quantitative polymerase chain reaction $(R T-q P C R)$. We extracted total RNA from the chondrocytes using TRIzol reagent (Invitrogen) according to the manufacturer's instructions. The purity of the total RNA was assessed with the A260/A280 ratio, and values of 1.8 and 2.0 were considered good. Total RNA was reverse transcribed into cDNA and amplified, and was subsequently quantified using RT-qPCR with SYBR-Green Ex Taq on a LightCycler 480 (Roche Applied Science, Indianapolis, IN, USA). Primers for ADAMTS5 were designed and obtained from Invitrogen. The glyceraldehyde 3-phosphate dehydrogenase (GAPDH) gene was used as a reference. The PCR conditions included 1 predenaturation cycle of $5 \mathrm{~min}$ at $95^{\circ} \mathrm{C}, 40-50$ cycles of $95^{\circ} \mathrm{C}$ for $30 \mathrm{sec}, 58-62^{\circ} \mathrm{C}$ for $30 \mathrm{sec}$, and $72^{\circ} \mathrm{C}$ for $30 \mathrm{sec}$, and a final extension at $72^{\circ} \mathrm{C}$ for $5 \mathrm{~min}$. Relative quantification was calculated using the $2^{-\Delta \Delta C \mathrm{CT}}$ method.

Bioinformatics analysis. Target genes of hsa-miR-15a were predicted by bioinformatics analysis using TargetScan 6.2 (http://www.targetscan.org) and/or microRNA.org (http:// www. microrna.org).

Luciferase reporter assay. The 3'-UTR of ADAMTS5 was PCR-amplified, cloned into the psiCHECK-2 vector and co-transfected with the control or hsa-miR-15a precursor into the chondrocytes. After $48 \mathrm{~h}$ of transfection, luciferase activity was evaluated using the Dual-luciferase activity assay system (DLR; Promega, Madison, WI, USA).

Measurement of DNA content. Purified total DNA was quantified using Quant-iT ${ }^{\text {TM }}$ PicoGreen ${ }^{\circledR}$ dsDNA reagent (Invitrogen) according to the manufacturer's instructions. Fluorescence was measured using a fluorescence microplate reader (Tecan Polarion, Stevenage, UK) at an excitation/emission wavelength of 480/520 nm. Lambda DNA (Sigma) was used as marker for determination of the DNA quantity.

Proteoglycan analysis. Total glycosaminoglycans (GAGs) were quantified using a 1,9-dimethylmethylene blue (DMMB) spectrophotometric analysis according to a previously described method (29). Briefly, following the addition of DMMB, the mixture was assayed for GAG at an absorbance of $525 \mathrm{~nm}$. Chondroitin sulfate C (Sigma) was used as a reference. 
Collagenase activity assay. After collecting the culture medium, collagenase activity assay was performed to determine the collagenase activity using an Enzchek Gelatinase/Collagenase assay kit (Invitrogen) according to the manufacturer's instructions. The assays were carried out in reaction buffer containing DQ Collagen Fluorescein conjugate at room temperature. Fluorescence was determined using a fluorescence microplate reader (SpectraMAX Gemini XS; Molecular Devices, Sunnyvale, CA, USA) at $485 \mathrm{~nm}$ excitation and $538 \mathrm{~nm}$ emission. Collagenase activity was determined using the formula provided with the assay kit and normalized to the weight of the explant. Collagenase type IV (Clostridium) was used as a reference standard.

Western blot analysis. Following culture for $24 \mathrm{~h}$, cell protein was extracted using protein lysis buffer. Protein concentration was assessed using the Bio-Rad DC protein assay kit (Bio-Rad, Ivrysur-Seine, France). The protein was separated by $10 \%$ sodium dodecyl sulfate-polyacrylamide gel electrophoresis (SDS-PAGE) and was then transferred to polyvinylidene difluoride (PVDF) membranes (Bio-Rad, Hercules, CA, USA). The membranes were blocked in a sealed in 5\% fresh non-fat dry milk for $2 \mathrm{~h}$ and were then incubated with the primary anti-type II collagen antibody (1:1,000 dilution; MAB1330; Chemicon, Millipore, Billerica, CA, USA) and primary anti-ADAMTS5 antibody (1:100 dilution; ab45042; Abcam, Cambridge, UK) for $2 \mathrm{~h}$ at room temperature or overnight at $4{ }^{\circ} \mathrm{C}$, followed with horseradish peroxidase-conjugated anti-mouse secondary antibody (accession numbers ab195239). An anti-GAPDH antibody (sc-365062; Santa Cruz Biotechnology, Inc., Santa Cruz, CA, USA) was used as a loading control. Finally, the samples were tested using enhanced chemiluminescence and densitometric analysis. For relative expression analysis, NIH Image $\mathbf{J}$ software (NIH Image, Bethesda, MD, USA) was performed to determine band intensity.

Statistical analysis. In the present study, the statistical package for the social sciences (SPSS) (version 17.0; SPSS Inc., Chicago, IL, USA) was used to perform statistical analysis. A student's t-test was performed to study statistical comparisons. A p-value $<0.05$ was considered to indicate a statistically significant difference.

\section{Results}

Expression of hsa-miR-15a is decreased in human OA chondrocytes. To dertermine the expression levels of hsa-miR-15a in human OA chondrocytes, we determined the levels of hsa-miR-15a in human OA cartilage specimens and normal cartilage tissues by RT-qPCR. As shown in Fig. 1, the expression level of hsa-miR-15a was significantly decreased in the OA chondrocytes compared with the normal cartilage tissue $(\mathrm{P}<0.05)$.

ADAMTS5 gene 3'-UTR carries a putative hsa-miR-15a binding site and is negatively regulated by hsa-miR-15a. To predict whether the ADAMTS5 gene mRNA 3'-UTR contains a hsa-miR-15a binding site, TargetScan 6.2 and microRNA.org were used to confirm the prediction. The results revealed that a binding site for hsa-miR-15a was in the ADAMTS5 mRNA 3'-UTR (Fig. 2A).

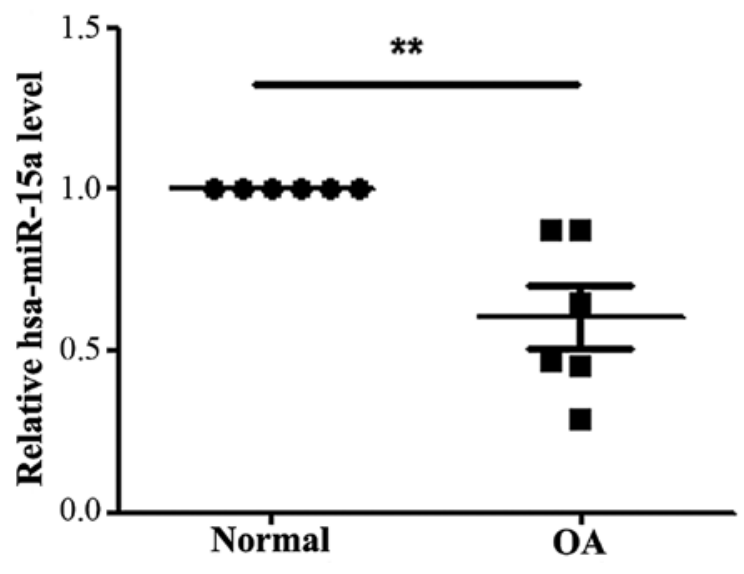

Figure 1. mRNA expression level of hsa-miR-15a in human osteoarthritic (OA) chondrocytes and normal chondrocytes. ${ }^{* *} \mathrm{p}<0.01$ compared with the normal control group.

In order to confirm that hsa-miR-15a binds to this region and leads to translational repression, we cloned the putative binding site into the pcDNA3/EGFP plasmid downstream of the EGFP reporter construct and co-transfected this plasmid into the cells with ASO-hsa-miR-15a or ASO-negative control (NC). We found that the relative EGFP intensity was significantly higher in the cells that were co-transfected with ASO-miR-15a compared with the cells co-transfected with ASO-NC. In addition, we cloned the putative binding site into pcDNA3/EGFP downstream of the EGFP reporter construct and co-transfected this plasmid with hsa-miR-15a mimics or NC. The results revealed that the relative EGFP intensity was significantly lower in the cells co-transfected with hsa-miR-15a mimics compared with those co-transfected with NC (Fig. 2B). Additionally, we performed RT-qPCR and western blot analysis to measure the mRNA and protein expression levels of ADAMTS5, respectively. Both RT-qPCR (Fig. 2C) and western blot analysis (Fig. 2D and E) revealed that the ADAMTS5 expression levels were significantly lower in the hsa-miR-15a mimics-transfected group compared with the NC-transfected group $(\mathrm{P}<0.05)$; however these levels were significantly higher in the ASO-miR-15a-transfected group compared with the ASO-NC-transfected group $(\mathrm{P}<0.05)$, respectively. These results suggest that hsa-miR-15a binds directly to the 3'-UTR of ADAMTS5 mRNA and inhibits gene expression. These results also indicate that ADAMTS5 is a direct target of hsa-miR-15a.

Downregulation of hsa-miR-15a expression decreases the aggregation of proteoglycan and increases the release of proteoglycan. The amount of proteoglycans was significantly decreased in the cell substrate generated by the OA chondrocytes transfected with ASO-miR-15a compared to the ASO-NCtransfected group (Fig. 3A), while the amount of proteoglycans was significantly increased in the culture medium compared to the ASO-NC-transfected group (Fig. 3B). However, as regards the total amount of proteoglycans, there were no significant differences observed between the ASO-miR-15a-transfected group and the controls (ASO-NC) (Fig. 3C). The total amount of proteoglycans was measured by combining the amount found in the cell substrate and that released into the medium at the end of the culture period. 

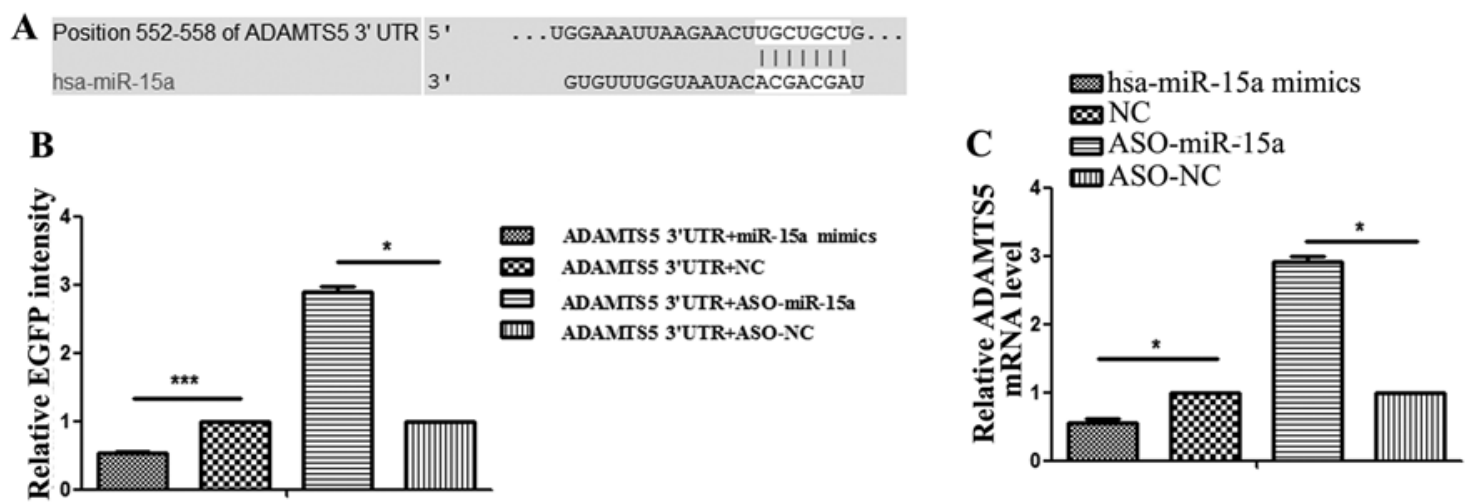

D

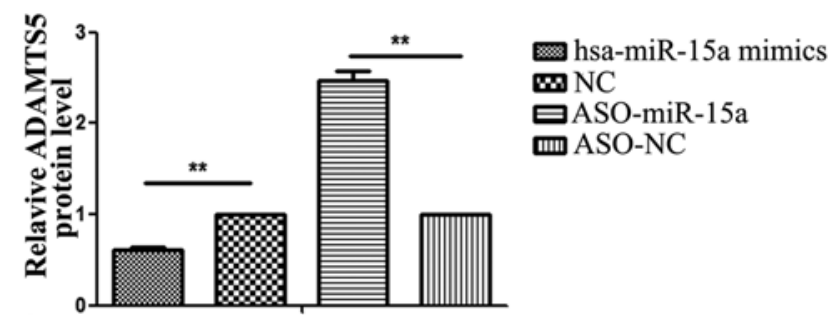

ADAMTS5 3'UTR+miR-15a mimics ADAMTS5 3'UTR+NC ADAMTS5 3'UTR+ASO-miR-15 ADAMIS5 3'UTR+ASO-NC

Figure 2. A disintegrin-like and metalloprotease (reprolysin type) with thrombospondin type 1 motif, 5 (ADAMTS5, also known as aggrecanase-2) gene 3'-UTR carries a putative hsa-miR-15a binding site and is negatively regulated by hsa-miR-15a. (A) Bioinformatics analysis predicted that ADAMTS5 is the target gene of hsa-miR-15a. (B) Luciferase reporter vector confirmed that hsa-miR-15a may bind directly to the 3'-UTR of ADAMTS5 mRNA. (C) Relative ADAMTS5 mRNA expression level regulated by hsa-miR-15a. (D) Relative ADAMTS5 protein expression level regulated by hsa-miR-15a. (E) Western blot analysis of ADAMTS5 expression regulated by hsa-miR-15a. ASO, antisense oligonucleotides; NC, negative control. ${ }^{*} \mathrm{p}<0.05,{ }^{* *} \mathrm{p}<0.01$ and ${ }^{* * * *} \mathrm{p}<0.001$ compared with the corresponding control group.
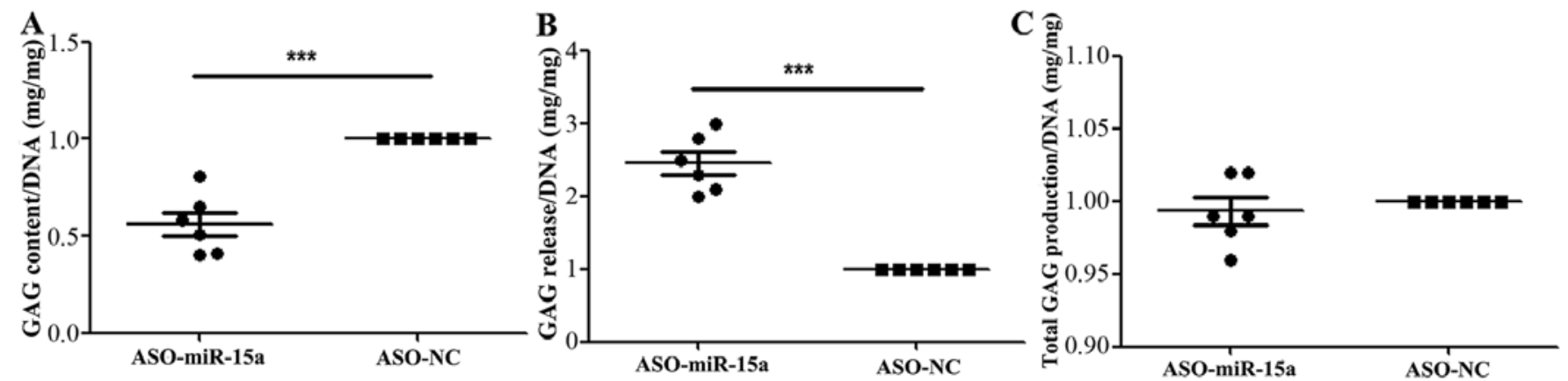

Figure 3. Low expression of hsa-miR-15a increases the release of proteoglycan and decreases the aggregation of proteoglycan. (A) Glycosaminoglycan (GAG) content in cell substrate is regulated by hsa-miR-15a. (B) GAG content in medium is regulated by hsa-miR-15a. (C) Total amount of GAG is regulated by hsa-miR-15a. ASO, antisense oligonucleotides; NC, negative control. ${ }^{* * *} \mathrm{p}<0.001$ compared with the ASO-NC group.

Downregulation of hsa-miR-15a expression decreases the aggregation of collagen and increases the release of collagen. The amount of collagen was significantly decreased in the cell substrate generated by the OA chondrocytes transfected with ASO-miR-15a compared to the ASO-NC-transfected group (Fig. 4A), while the amount of collagen was significantly increased in the culture medium of the cells transfected with ASO-miR-15a compared to the ASO-NC-transfected group (Fig. 4B). In addition, the total amount of collagen was significantly decreased in the ASO-miR-15a-transfected group compared with the controls (ASO-NC) (Fig. 4C). However, collagenase activity was significantly increased in ASO-miR-15a-transfected group compared with the controls (Fig. 4D). Moreover, the protein expression of collagen II was significantly lower $(0.75 \pm 0.14$ vs. $1.88 \pm 0.16$, with a $40 \%$ decrease) in the ASO-miR-15a-transfected group compared with the ASO-NC-transfected group (Fig. 4E). These results indicate that the decreased expression of hsa-miR-15a inhibits the aggregation of collagen, while the overexpression of hsa-miR-15a promotes the aggregation of collagen.

Downregulation of ADAMTS5 increases the aggregation of proteoglycan and decreases the release of proteoglycan. We performed RT-qPCR to measure the expression levels of ADAMTS5 in human OA chondrocytes, as well as in normal cartilage tissue. As shown in Fig. 5A, the expression level of ADAMTS5 was significantly increased in the OA chondrocytes compared with the normal cartilage tissue $(\mathrm{P}<0.05)$. 

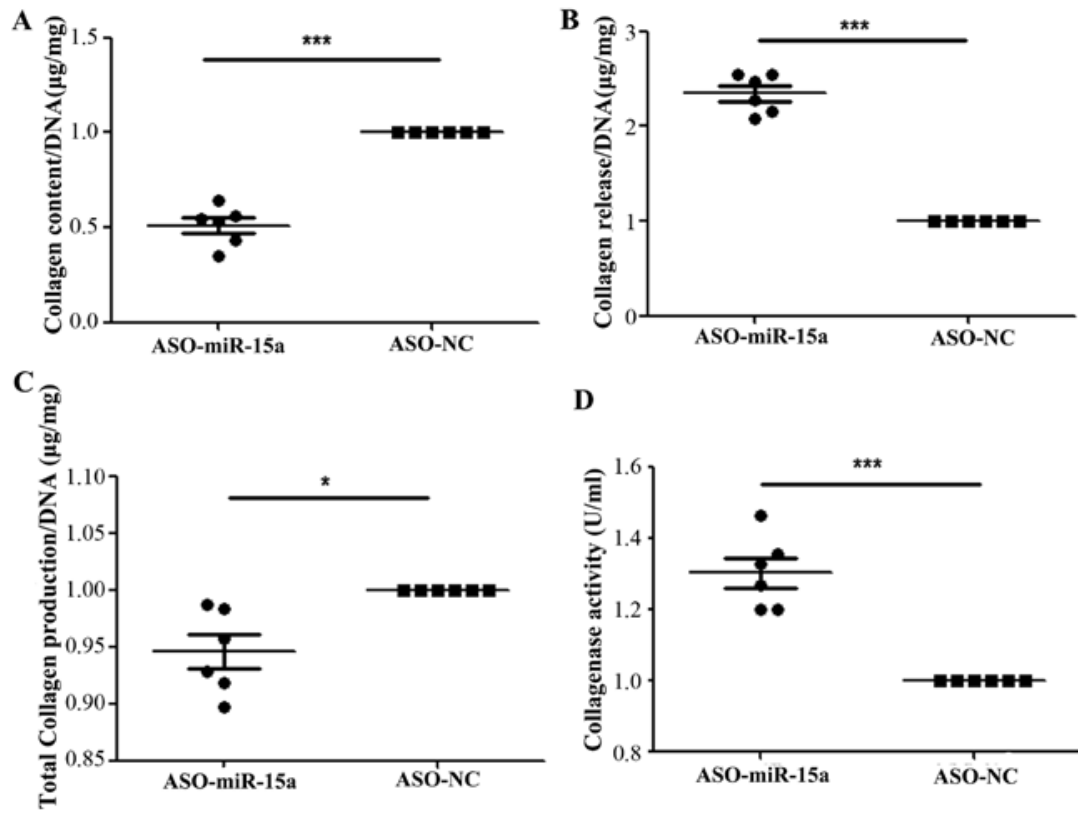

D
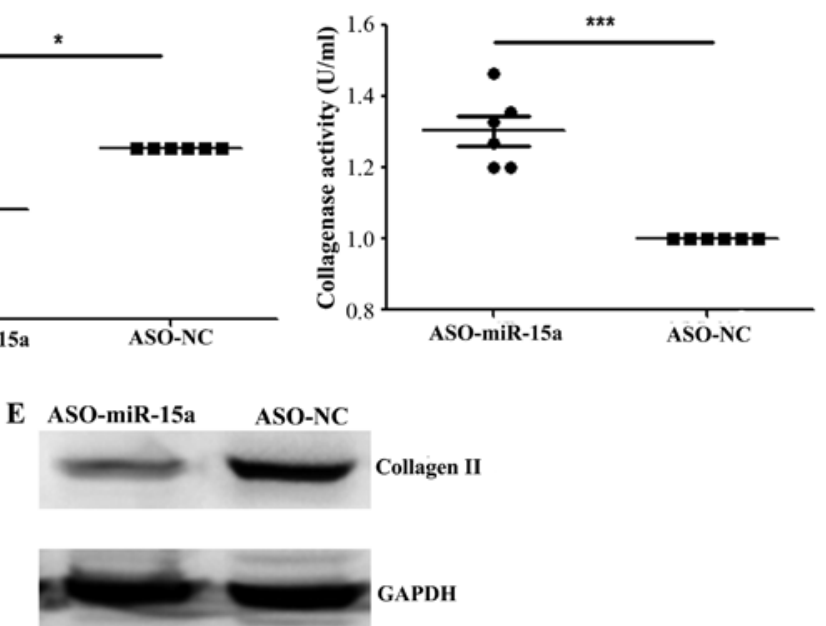

Figure 4. Low expression of hsa-miR-15a increases the release of collagen and decreases the aggregation of collagen. (A) Collagen content in cell substrate is regulated by hsa-miR-15a. (B) Collagen content in medium is regulated by hsa-miR-15a. (C) Total amount of collagen is regulated by hsa-miR-15a. (D) Collagenase activity is regulated by hsa-miR-15a. (E) Western blot analysis of collagen II regulated by hsa-miR-15a. ASO, antisense oligonucleotides; NC, negative control. " $\mathrm{p}<0.05$ and ${ }^{* * *} \mathrm{p}<0.001$ compared with the ASO-NC group.
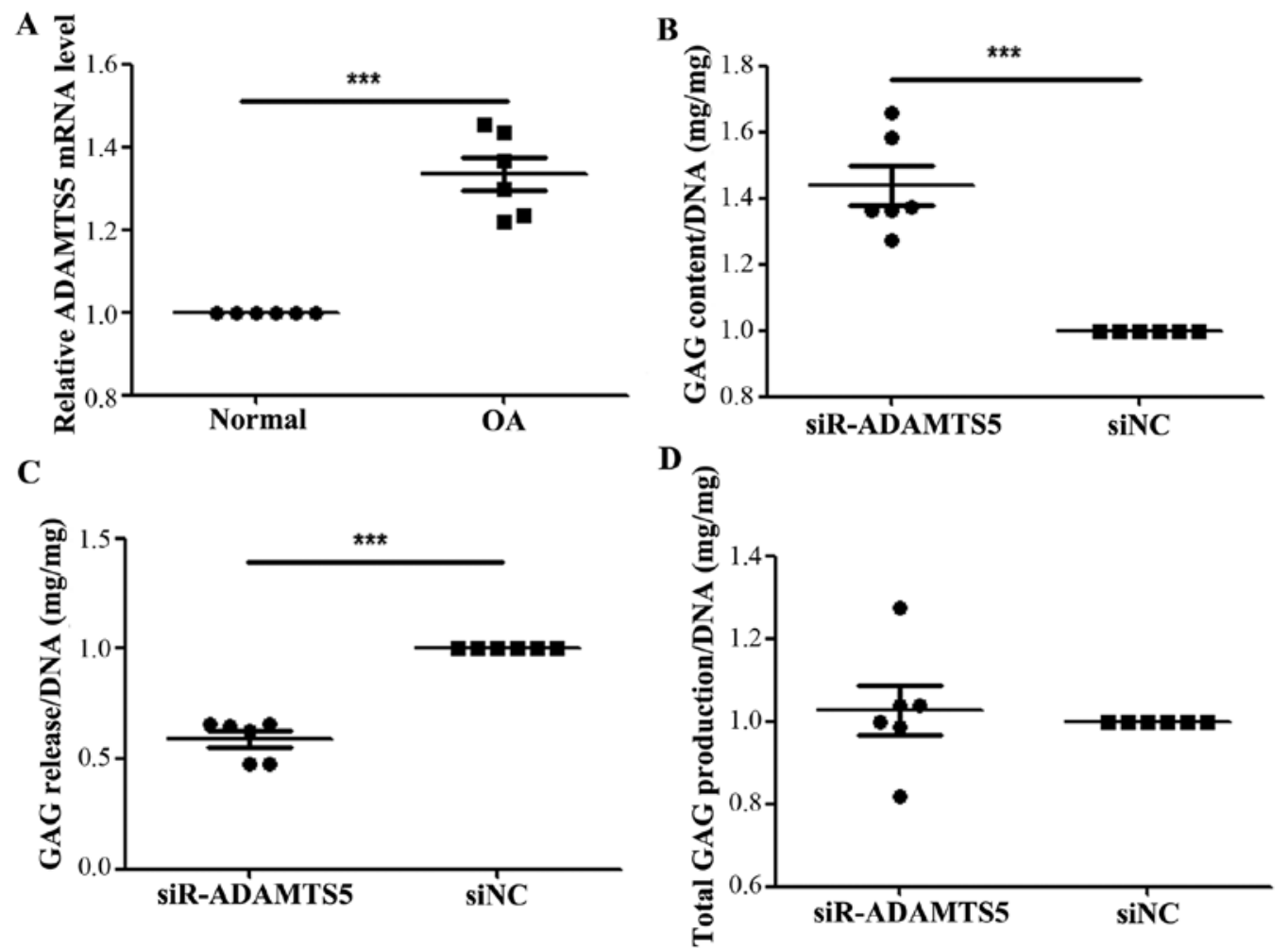

Figure 5. A disintegrin-like and metalloprotease (reprolysin type) with thrombospondin type 1 motif, 5 (ADAMTS5, also known as aggrecanase-2) decreases the aggregation of proteoglycan and increases the release of proteoglycan. (A) Relative ADAMTS5 mRNA expression in human osteoarthritic (OA) chondrocytes and normal chondrocytes. (B) Glycosaminoglycan (GAG) content in cell substrate is regulated by ADAMTS5. (C) GAG content in medium is regulated by ADAMTS5. (D) Total amount of is GAG regulated by ADAMTS5. ASO, antisense oligonucleotides; NC, negative control. ${ }^{* * *}$ p $<0.001$ compared with the siNC group. 

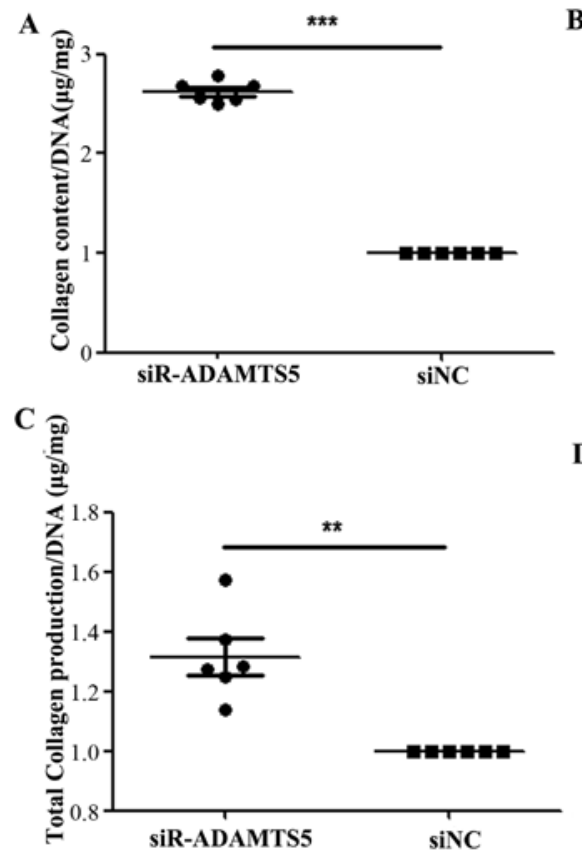

E siR-ADAMTS5 siNC

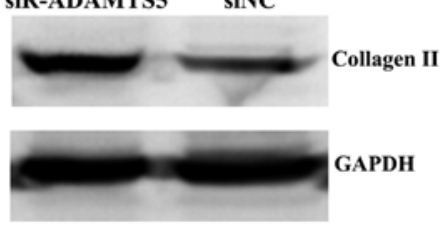

B

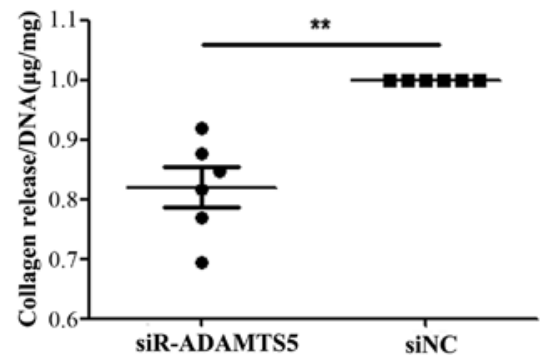

)

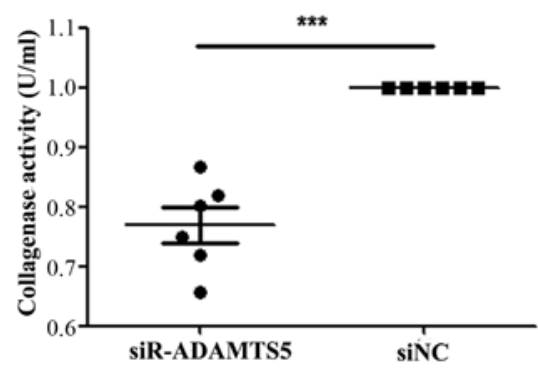

Figure 6. A disintegrin-like and metalloprotease (reprolysin type) with thrombospondin type 1 motif, 5 (ADAMTS5, also known as aggrecanase-2) decreases the aggregation of collagen and increases the release of collagen. (A) Collagen content in cell substrate is regulated by ADAMTS5. (B) Collagen content in medium is regulated by ADAMTS5. (C) Total amount of collagen is regulated by ADAMTS5. (D) Collagenase activity is regulated by ADAMTS5. (E) Western blot analysis of collagen II regulated by ADAMTS5. ASO, antisense oligonucleotides; NC, negative control. ${ }^{* *} \mathrm{p}<0.01$ and ${ }^{* * * *} \mathrm{p}<0.001$ compared with the siNC group.

To confirm a more definitive function of ADAMTS5 in the development of OA, we used ADAMTS5 siRNA to transfect human OA chondrocytes. The results revealed that the amount of proteoglycan was significantly increased in the chondrocyte substrate of the siR-ADAMTS5 group compared with that of the silenced negative control (siNC) group (Fig. 5B), whereas the amount released into the medium was significantly decreased compared with siNC group (Fig. 5C). No significant differences were observed in the total amount of proteoglycans between the 2 groups (Fig. 5D).

Downregulation of ADAMTS5 increases the aggregation of collagen and decreases the release of collagen. The amount of collagen was significantly increased in the chondrocyte substrate of the siR-ADAMTS5 group compared with that of the siNC group (Fig. 6A), whereas the amount released into the medium was significantly decreased compared with the siNC group (Fig. 6B). In addition, the total amount of collagen was significantly increased in the siR-ADAMTS5 group compared with the siNC group (Fig. 6C), while the collagenase activity was significantly lower in the siR-ADAMTS5 group than that in the siNC group (Fig. 6D). Furthermore, the protein expression of collagen II was significantly higher $(2.22 \pm 0.04$ vs $1.04 \pm 0.02$, with a $50 \%$ increase) in the siR-ADAMTS5 group compared with the siNC group (Fig. 6E).

\section{Discussion}

miRNAs are involved in normal skeletal development (30), and their levels are associated with the pathogenesis of degenerative disorders, such as OA $(31,32)$. Hence, it is necessary to elucidate the function of miRNA in the OA. In the present study, we examined the expression and role of hsa-miR-15a in the pathogenesis of OA. We found that hsa-miR-15a was expressed at lower levels in OA chondrocytes compared with normal healthy control tissue, suggesting that hsa-miR-15a plays an important role in the pathogenesis of OA. Using TargetScan6.2 and microRNA.org to screen the targets of hsa-miR-15, ADAMTS5 was predicted to be a target of hsa-miR-15a, and the binding site was found in the ADAMTS5 gene 3'-UTR. Moreover, the expression of ADAMTS5 was found to be negatively regulated by hsa-miR-15a. In addition, ADAMTS5, as a key enzyme in $\mathrm{OA}$, has been reported to play a role in the degradation of articular cartilage (24). Thus, we hypothesized that hsa-miR-15a may exert protective effects against OA through the negative regulation of the expression of ADAMTS5.

In order to prove this hypothesis, we first determined the expression level of has-miR-15a in both OA and normal chondrocytes. As previously mentioned, the expression level of hsa-miR-15a was significantly decreased in the OA chondrocytes compared with the normal chondrocytes. 
Subsequently, we further assessed the prediction revealed by bioinformatics analysis that ADAMTS5 is targeted by hsa-miR-15a. Luciferase reporter assay confirmed the prediction, and demonstrated that ADAMTS5 is directly targeted by hsa-miR-15a at its 3'-UTR. In addition, we also determined the expression mRNA and protein levels of ADAMTS5 when the level of hsa-miR-15a had been altered. By examining the overexpression (by transfection with hsa-miR-15a mimics) or downregulation (by transfection with ASO-hsa-miR-15a) of the level of hsa-miR-15a, we found that the expression level of ADAMTS5 inversely correlated with hsa-miR-15a.

As a key enzyme in OA, in this study, ADAMTS5 was found to be markedly increased in OA chondrocytes, which was in line with the findings of previous studies $(22,33,34)$. ADAMTS5 (namely aggrecanase-2) are members of the ADAMTS family. Together with ADAMTS4 (namely aggrecanase-1), they have the ability to cleave the aggrecan core protein $(35,36)$, resulting in the destruction of cartilage function, which is subsequently followed by irreversible collagen degradation $(22,37,38)$. Previous research has confirmed that ADAMTS4 and ADAMTS5 are the most efficient aggrecanases in vitro, but ADAMTS5 presents more actively than ADAMTS4, and rather than ADAMTS4, ADAMTS5 participates in the early stages of development of OA $(33,39)$. Moreover, the deletion of ADAMTS5 protects against OA by decreasing aggrecan degradation (40). Additionally, the intervention of aggrecanases has been considered as an effective therapeutic method for treatment of OA (37). In the present study, we demonstrated the important role of ADAMTS5 in the pathogenesis of OA. To confirm the function of ADAMTS5, we further silenced the expression of ADAMTS5. Our results indicated that the aggregation of proteoglycan and the collagen content were markedly increased, but the release of proteoglycan and collagen was significantly decreased following the suppression of ADAMTS5. Moreover, the total collagen production was significantly higher, and collagenase activity was markedly lower in the siR-ADAMTS5-transfected group compared with the siNC-transfected group. However, the silencing of ADAMTS5 did not have a marked effect on the total proteoglycan production, indicating that diminished breakdown induced the increased retention of proteoglycan content, rather than the synthetic activity of proteoglycan being increased. These results are in accordance with those of a previous study conducted by Vonk et al (41), which suggests that ADAMTS5 plays an important role in the pathogenesis of OA through the inhibition of the aggregation of proteoglycan and collagen content and the increase in the collagenase activity.

The expression of hsa-miR-15a was downregulated in this study to confirm the protective effects of hsa-miR-15a against OA. Opposite effects were observed in the amount of proteoglycan and collagen in the cellular matrix and medium, the expression level of collagen II, and collagenase activity compared with the effects revealed by the suppression of ADAMTS5 expression. Our results demonstrated that the aggregation of proteoglycan and collagen content were significantly decreased, but the release of proteoglycan and collagen was markedly increased in the ASO-miR-15a-transfected compared with the normal group (ASO-NC). Moreover, the total collagen production was significantly lower, and collagenase activity was markedly higher following the downregulation of miR-15a.
These data suggest that hsa-miR-15a exerts protective effects against OA by suppressing the expression of ADAMTS5.

In conclusion, to the best of our knowledge, this study provides the first evidence that hsa-miR-15 plays a protective role in OA by directly targeting ADAMTS5. Our results provide significant clinical evidence for future studies investigating the beneficial effects of targeting ADAMTS5 as a biological method for the treatment of OA.

\section{References}

1. Blalock D, Miller A, Tilley M and Wang J: Joint instability and osteoarthritis. Clin Med Insights Arthritis Musculoskelet Disord 8: 15-23, 2015.

2. Lee AS, Ellman MB, Yan D, Kroin JS, Cole BJ, van Wijnen AJ and Im HJ: A current review of molecular mechanisms regarding osteoarthritis and pain. Gene 527: 440-447, 2013.

3. Loeser RF, Goldring SR, Scanzello CR and Goldring MB: Osteoarthritis: a disease of the joint as an organ. Arthritis Rheum 64: 1697-1707, 2012.

4. Pereira D, Peleteiro B, Araujo J, Branco J, Santos RA and Ramos E: The effect of osteoarthritis definition on prevalence and incidence estimates: a systematic review. Osteoarthritis and cartilage/OARS. Osteoarthritis Res Soc 19: 1270-1285, 2011.

5. Zhang Y, Jia J, Yang S, Liu X, Ye S and Tian H: MicroRNA-21 controls the development of osteoarthritis by targeting GDF-5 in chondrocytes. Exp Mol Med 46: e79, 2014.

6. Litwic A, Edwards MH, Dennison EM and Cooper C: Epidemiology and burden of osteoarthritis. Br Med Bull 105: 185-199, 2013.

7. Buckwalter JA, Saltzman C and Brown T: The impact of osteoarthritis: implications for research. Clin Orthop Relat Res 427 (Suppl): S6-S15, 2004

8. Wieland HA, Michaelis M, Kirschbaum BJ and Rudolphi KA: Osteoarthritis - an untreatable disease? Nat Rev Drug Discov 4: 331-344, 2005.

9. Flower RJ: The development of COX2 inhibitors. Nat Rev Drug Discov 2: 179-191, 2003.

10. Goldring MB and Marcu KB: Epigenomic and microRNAmediated regulation in cartilage development, homeostasis, and osteoarthritis. Trends Mol Med 18: 109-118, 2012.

11. Wu C, Tian B, Qu X, Liu F, Tang T, Qin A, Zhu Z and Dai K: MicroRNAs play a role in chondrogenesis and osteoarthritis (Review). Int J Mol Med 34: 13-23, 2014.

12. Le LT, Swingler TE and Clark IM: Review: The role of microRNAs in osteoarthritis and chondrogenesis. Arthritis Rheum 65: 1963-1974, 2013.

13. Bartel DP: MicroRNAs: genomics, biogenesis, mechanism, and function. Cell 116: 281-297, 2004.

14. Sethupathy P and Collins FS: MicroRNA target site polymorphisms and human disease. Trends Genet 24: 489-497, 2008.

15. Djuranovic S, Nahvi A and Green R: miRNA-mediated gene silencing by translational repression followed by mRNA deadenylation and decay. Science 336: 237-240, 2012.

16. Seeliger C1, Karpinski K, Haug AT, Vester H, Schmitt A, Bauer JS and van Griensven M: Five freely circulating miRNAs and bone tissue miRNAs are associated with osteoporotic fractures. J Bone Miner Res 29: 1718-1728, 2014.

17. Iliopoulos D, Malizos KN, Oikonomou P and Tsezou A: Integrative microRNA and proteomic approaches identify novel osteoarthritis genes and their collaborative metabolic and inflammatory networks. PLoS One 3: e3740, 2008.

18. Swingler TE, Wheeler G, Carmont V, Elliott HR, Barter MJ, Abu-Elmagd M, Donell ST, Boot-Handford RP, Hajihosseini MK, Münsterberg A, et al: The expression and function of microRNAs in chondrogenesis and osteoarthritis. Arthritis Rheum 64: 1909-1919, 2012.

19. Yu C, Chen WP and Wang XH: MicroRNA in osteoarthritis. J Int Med Res 39: 1-9, 2011.

20. Dudek KA, Lafont JE, Martinez-Sanchez A and Murphy CL: Type II collagen expression is regulated by tissue-specific miR-675 in human articular chondrocytes. J Biol Chem 285: 24381-24387, 2010.

21. Tardif G, Hum D, Pelletier JP, Duval N and Martel-Pelletier J: Regulation of the IGFBP-5 and MMP-13 genes by the microRNAs miR-140 and miR-27a in human osteoarthritic chondrocytes. BMC Musculoskelet Disord 10: 148, 2009. 
22. Bondeson J, Wainwright S, Hughes C and Caterson B: The regulation of the ADAMTS4 and ADAMTS5 aggrecanases in osteoarthritis: a review. Clin Exp Rheumatol 26: 139-145, 2008.

23. Little $\mathrm{CB}$ and Fosang AJ: Is cartilage matrix breakdown an appropriate therapeutic target in osteoarthritis - insights from studies of aggrecan and collagen proteolysis? Curr Drug Targets 11: 561-575, 2010.

24. Glasson SS, Askew R, Sheppard B, Carito B, Blanchet T, Ma HL, Flannery CR, Peluso D, Kanki K, Yang Z, et al: Deletion of active ADAMTS5 prevents cartilage degradation in a murine model of osteoarthritis. Nature 434: 644-648, 2005.

25. Matsukawa T, Sakai T, Yonezawa T, Hiraiwa H, Hamada T, Nakashima M, Ono Y, Ishizuka S, Nakahara H, Lotz MK, et al: MicroRNA-125b regulates the expression of aggrecanase-1 (ADAMTS-4) in human osteoarthritic chondrocytes. Arthritis Res Ther 15: R28, 2013

26. Altman R, Asch E, Bloch D, Bole G, Borenstein D, Brandt K, Christy W, Cooke TD, Greenwald R, Hochberg M, et al: Development of criteria for the classification and reporting of osteoarthritis. Classification of osteoarthritis of the knee. Diagnostic and Therapeutic Criteria Committee of the American Rheumatism Association. Arthritis Rheum 29: 1039-1049, 1986.

27. Lafont JE, Talma S and Murphy CL: Hypoxia-inducible factor 2alpha is essential for hypoxic induction of the human articular chondrocyte phenotype. Arthritis Rheum 56: 3297-3306, 2007.

28. Zhang XJ, Ye H, Zeng CW, He B, Zhang H and Chen YQ: Dysregulation of miR-15a and miR-214 in human pancreatic cancer. J Hematol Oncol 3: 46, 2010.

29. Pieper JS, van der Kraan PM, Hafmans T, Kamp J, Buma P, van Susante JL, van den Berg WB, Veerkamp JH and van Kuppevelt TH: Crosslinked type II collagen matrices: Preparation, characterization, and potential for cartilage engineering. Biomaterials 23: 3183-3192, 2002.

30. Papaioannou G, Lisse T and Kobayashi T: miRNAs in bone formation and homeostasis. In: microRNA in Regenerative Medicine. Sen CK (ed). Elsevier Inc., London, pp350-380, 2015.

31. Miyaki S and Asahara $\mathrm{H}$ : Macro view of microRNA function in osteoarthritis. Nat Rev Rheumatol 8: 543-552, 2012.

32. Beyer C, Zampetaki A, Lin NY, Kleyer A, Perricone C, Iagnocco A, Distler A, Langley SR, Gelse K, Sesselmann S, et al: Signature of circulating microRNAs in osteoarthritis. Ann Rheum Dis 74: e18, 2015 .
33. Gendron C, Kashiwagi M, Lim NH, Enghild JJ, Thøgersen IB, Hughes C, Caterson B and Nagase H: Proteolytic activities of human ADAMTS-5: comparative studies with ADAMTS-4. J Biol Chem 282: 18294-18306, 2007.

34. Thirunavukkarasu K, Pei Y and Wei T: Characterization of the human ADAMTS-5 (aggrecanase-2) gene promoter. Mol Biol Rep 34: 225-231, 2007.

35. Abbaszade I, Liu RQ, Yang F, Rosenfeld SA, Ross OH, Link JR, Ellis DM, Tortorella MD, Pratta MA, Hollis JM, et al: Cloning and characterization of ADAMTS11, an aggrecanase from the ADAMTS family. J Biol Chem 274: 23443-23450, 1999.

36. Tortorella MD, Burn TC, Pratta MA, Abbaszade I, Hollis JM, Liu R, Rosenfeld SA, Copeland RA, Decicco CP, Wynn R, et al: Purification and cloning of aggrecanase-1: a member of the ADAMTS family of proteins. Science 284: 1664-1666, 1999.

37. Verma P and Dalal K: ADAMTS-4 and ADAMTS-5: Key enzymes in osteoarthritis. J Cell Biochem 112: 3507-3514, 2011.

38. Song RHD, Tortorella MD, Malfait AM, Alston JT, Yang Z, Arner EC and Griggs DW: Aggrecan degradation in human articular cartilage explants is mediated by both ADAMTS- 4 and ADAMTS-5. Arthritis Rheum 56: 575-585, 2007.

39. Stanton H, Rogerson FM, East CJ, Golub SB, Lawlor KE, Meeker CT, Little CB, Last K, Farmer PJ, Campbell IK, et al: ADAMTS5 is the major aggrecanase in mouse cartilage in vivo and in vitro. Nature 434: 648-652, 2005.

40. Majumdar MK, Askew R, Schelling S, Stedman N, Blanchet T, Hopkins B, Morris EA and Glasson SS: Double-knockout of ADAMTS-4 and ADAMTS-5 in mice results in physiologically normal animals and prevents the progression of osteoarthritis. Arthritis Rheum 56: 3670-3674, 2007.

41. Vonk LA, Kragten AH, Dhert WJ, Saris DB and Creemers LB: Overexpression of hsa-miR-148a promotes cartilage production and inhibits cartilage degradation by osteoarthritic chondrocytes. Osteoarthritis and cartilage/OARS. Osteoarthritis Res Soc 22: $145-153,2014$. 